\title{
Study on expression of plasma sCD138 in patients with hemorrhagic fever with renal syndrome
}

\author{
Jing $\mathrm{Li}^{\dagger}$, Hong Du${ }^{\dagger}$, Xue-Fan Bai, Xiao-Yan Wang, Ying Zhang, Hong Jiang ${ }^{*}$ and Ping-Zhong Wang ${ }^{*}$
}

\begin{abstract}
Background: Until now, there is non-specific treatment, and exploring early and novel biomarkers to determine the disease severity and prognosis of hemorrhagic fever with renal syndrome (HFRS) would be of importance for clinician to take systematic and timely intervention. This study observed the expression of plasma SCD138, a soluble component shedding from the glycocalyx (GCX) to the circulating blood, and evaluated its predictive value on disease severity and prognosis of HFRS.
\end{abstract}

Methods: One hundred and seventy-six patients with HFRS who were treated at our center between January 2011 and December 2013 were randomly enrolled in this study. The patients were divided into a mild-type group, a moderate-type group, a severe-type group and a critical-type group according to the HFRS criteria for clinical classification. Thirty-five blood samples from healthy subjects were obtained as the controls. The concentrations of sCD138 were detected using enzyme linked immunosorbent assay (ELISA). The levels of prothrombin time (PT), activated partial thromboplastin time (APTT), fibrinogen (Fib), albumin (ALB), alanine aminotransferase (ALT), aspartate aminotransferase (AST), white blood cells $(\mathrm{WBC})$, platelets (PLT), glucose (GLU), blood urea nitrogen (BUN) and serum creatinine $(\mathrm{SCr})$ in the samples were routinely tested. The levels of sCD138 among the different types were compared; the correlation among SCD138 and the laboratory parameters mentioned above were analyzed. The predictive effectiveness for prognosis of SCD138 was evaluated using the receiver operating characteristic (ROC) curve analysis.

Results: Except for the mild-type, the levels of sCD138 in the moderate-, severe- and critical-type patients during the acute stage were significantly higher than that of the convalescent stage and the control $(P<0.05)$. With the aggravation of the disease, the levels of sCD138 during the acute stage had an increasing tendency, while demonstrated no significant difference among the moderate-, severe- and critical-type patients ( $P>0.05)$. SCD138 was negatively correlated with Fib, PLT and $A L B$, and was positively correlated with WBC and AST $(P<0.05)$. SCD138 demonstrated predictive effectiveness for prognosis with the area under the curve (AUC) of $0.778(P<0.001)$.

Conclusion: Dynamic detection of plasma sCD138 might be benefit to evaluating the disease severity and prognosis of the patients with HFRS.

Keywords: Hantavirus, Hemorrhagic fever with renal syndrome, Soluble CD138, Prognosis

\footnotetext{
*Correspondence: jiangh518@126.com; wangpz63@126.com

${ }^{\dagger}$ Equal contributors

Center of Infectious Diseases, Tangdu Hospital, Air-force Military Medical

University, 569 Xinsi Rd, Baqiao District, Xi'an, Shaanxi 710038, China
} 


\section{Background}

As a rodent-borne disease caused by Hantavirus, hemorrhagic fever with renal syndrome (HFRS) usually manifest fever, hypotension, hemorrhage and kidney injury $[1,2]$. Like sepsis, HFRS has general pathophysiologic characteristics of systemic inflammatory response syndrome (SIRS), while it also has unique clinical progresses usually through febrile, hypotensive, oliguric, diuretic and convalescent phases [3, 4]. In some critical-type HFRS, the febrile, hypotensive and oliguric phases can overlap, resulting in refractory shock, acute respiratory distress syndrome (ARDS), acute kidney injury (AKI), encephalopathy, severe blood clotting dysfunction and multiple organ dysfunction syndrome (MODS) [5].

Until now, there is non-specific treatment, and early discovery, diagnosis, monitoring and supportive management are still the major treatment principle of the disease [6]. Considering the evaluative and predictive ability of the disease severity of HFRS on laboratory parameters routinely tested is still poor because of the complicated pathophysiology, exploring early and novel biomarkers to determine the disease severity and prognosis would be of importance for clinician to take systematic and timely intervention [7].

Glycocalyx (GCX) plays an important role on regulating vasopermeability, promoting platelet aggregation and coagulation disturbance as a major ingredient of endothelial surface layer (ESL) [8], and the degree of GCX component degradation can reflect the degree of neutrophil adhesion, endothelial dysfunction, tissue injury and severity of sepsis [9]. Glucoprotein and proteoglycans (PGs) are principal components of GCX, and PGs has a core of protein, which can connect with negative charge GAG side chain [10]. Syndecans family are major components of PGs, including the CD138, also called syndecan-1, is composed of a ectodomain, a single membrane-spanning domain and a short endochylema domain with phosphorylated site [11, 12]. Under physiopathologic conditions, such as sepsis, chronic inflammation, acute decompensated heart failure and ischemical reperfusion injury [13-16], syndecan-1 can be shed from the GCX to the circulating blood as soluble type, which can be considered as an potential marker on predicting prognosis and diagnosis $[17,18]$.

As far as we know, there was no research on exploring the expression and relationship between soluble components of PGs and disease severity and prognosis of HFRS. In this observational prospective study, we observed the expression of plasma sCD138 in patients with HFRS and explore its predictive capacity for disease severity and prognosis.

\section{Methods}

\section{Study participants}

One hundred and seventy-six patients with HFRS that were treated at our center between January 2011 and
December 2013 were randomly enrolled in this study. The demographic characteristics of the patients were collected from medical records. Patients who had diabetes, autoimmune disease, kidney diseases, hematological disease, cardiovascular disease, viral hepatitis and other infectious diseases such as sepsis infected by bacteria and fungus, dengue hemorrhagic fever, malaria, et al., were excluded.

The diagnosis of HFRS was made based on the positive enzyme linked immunosorbent assay (ELISA) result for both specific IgM and IgG antibodies against Hantaan virus (HTNV), an sole serotype epidemic in the study region as far as we know, in serum of the acute phase. The assay was performed using IgG/IgM capture ELISA kits and was analyzed via a multifunctional autoanalyzer (BIORAD-680, United States).

According to the HFRS criteria of clinical classification [19], the severity of HFRS was classified into four types: (1) mild, defined as patients who had kidney injury without oliguria and hypotension; (2) moderate, defined as patients who had uremia, effusion (bulbar conjunctiva), hypotension, hemorrhage (skin and mucous membranes), and AKI with typical oliguria; (3) severe, defined as patients who had severe uremia, effusion (bulbar conjunctiva and either peritoneum or pleura), hemorrhage (skin and mucous membranes), hypotension and AKI with oliguria (urine output of $50-500 \mathrm{~mL} /$ day) for $\leq 5$ days or anuria (urine output of $<100 \mathrm{~mL} /$ day) for $\leq 2$ days; and (4) critical, defined as patients who usually had one or more of the following complications compared with the severe patients: refractory shock ( $\geq 2$ days), visceral hemorrhage, heart failure, pulmonary edema, brain edema, severe secondary infection, and severe AKI with oliguria (urine output of $50-500 \mathrm{~mL} /$ day) for > 5 days or anuria (urine output of $<100 \mathrm{~mL} /$ day) for $>$ 2 days. Considering the clinical conditions that a majority of the survival patients had been discharged before the convalescent phase and the degree of AKI that was still severe during the early stage of the diuretic phase, the acute stage was defined as the period that included the febrile, hypotensive, and oliguric phases and the early three days of the diuretic phase in this study, and the convalescent stage was defined as the diuretic and convalescent phase except the early three days of the diuretic phase. Furthermore, the patients received follow up until 28 days after discharge, and the prognosis (death) in this study was defined as patient death during hospitalization or within the 28 days following discharge.

\section{Blood samples and detection}

Three hundred and thirteen venous blood samples were drawn randomly from the patients including 176 samples during the acute stage, and 137 samples during the convalescent stage. Thirty-five blood samples from healthy subjects were obtained as the controls. All of the samples 
Table 1 Demographic characteristics for patients with HFRS

\begin{tabular}{llllll}
\hline & $\begin{array}{l}\text { Mild group } \\
(n=32)\end{array}$ & $\begin{array}{l}\text { Moderate group } \\
(n=50)\end{array}$ & $\begin{array}{l}\text { Severe group } \\
(n=43)\end{array}$ & $\begin{array}{l}\text { Critical group } \\
(n=51)\end{array}$ & $\begin{array}{l}\text { Control group } \\
(n=35)\end{array}$ \\
\hline${ }^{\mathrm{a}}$ Female, $\mathrm{n}(\%)$ & $8(25.0)$ & $12(24.0)$ & $8(18.6)$ & $12(23.5)$ & $11(31.4)$ \\
$\mathrm{b}$ Age, years & $40.27 \pm 13.72$ & $41.42 \pm 13.23$ & $41.24 \pm 12.61$ & $48.58 \pm 11.56$ & $41.15 \pm 12.24$ \\
\hline
\end{tabular}

aPearson's $X^{2}$ test: $X^{2}=1.757, P=0.780$

${ }^{\mathrm{b}}$ ANOVA: $\mathrm{F}=2.428, P=0.072$

were stored in ethylenediamine tetraacetic acid (EDTA) tubes and were centrifuged at $2500 \mathrm{rpm}$ for $10 \mathrm{~min}$ at $4^{\circ}$ $\mathrm{C}$ within $2 \mathrm{~h}$ after drawing. The plasma supernatant was pipetted carefully and transferred to polypropylene tubes and then stored at $-80{ }^{\circ} \mathrm{C}$ prior to analysis.

sCD138 levels were measured with commercially available ELISA kits (Quantikine, XiTang, Inc., Shanghai, China) and were tested using a multifunctional autoanalyzer (BIORAD-680, United States) according to the manufacturer's instructions. Each sample was detected in duplicate. According to the difference of the clinical typing, some sample of the patients were further taken for multiple dilutions and a reasonable detection result was finally selected.

Eleven laboratory parameters including prothrombin time (PT), activated partial thromboplastin time (APTT), fibrinogen (Fib), albumin (ALB), alanine aminotransferase (ALT), aspartate aminotransferase (AST), white blood cells (WBC), platelets (PLT), glucose (GLU), blood urea nitrogen $(\mathrm{BUN})$ and serum creatinine $(\mathrm{Scr})$ were routinely tested using autoanalyzers (Sysmex XT-4000i, Japan; Hitachi 7600-100, Japan; hemagglutination analyzers PUN-2048B, Sysmex, Japan; blood gas analyzer, GEM Premier 3500). All the laboratory parameters mentioned above and $\mathrm{sCD} 138$ were measured in the same time frame.

\section{Statistical analysis}

Statistical analysis was performed using SPSS 17.0 software (SPSS Inc., Chicago, IL, USA). Tables were created using Excel 2003 (Microsoft), and figures were created using GraphPad Prism 5 (GraphPad Software, SanDiego CA). Continuous variables are presented as the mean \pm $\mathrm{SD}$ and were analyzed by Kolmogorov-Smirnov's test for normal distribution and by Levene's test for the homogeneity of variance. The variables among the four types were compared by the SNK test for normally distributed variables. The non-normally distributed variables are presented as medians with interquartile ranges and were compared by the non-parametric Kruskal-Wallis $\mathrm{H}$ test. The Nemenyi Rank test was used to compare the differences among the four types. The frequencies and percentages are given for qualitative variables, and the differences among the four types were tested using the Pearson's chi-square test. Spearman's correlation coefficient was used to determine the relationship between sCD138 and the laboratory parameters as mentioned above. The predictor values of sCD138 for disease prognosis were tested using receiver operating characteristic (ROC) curves and quantified by calculating the area under the ROC curve (AUC) and the 95\% confidence interval (CI). A two-tailed $P<0.05$ was considered statistically significant.

\section{Ethics statement}

The study was approved by the Institutional Review Board of Tangdu Hospital. Before inclusion, the patients were informed about the objectives of this study, and they or their direct relatives agreed and signed the informed consent form before inclusion.

\section{Results}

Clinical typing and demographic characteristics for patients with HFRS

Of the enrolled patients, including 40 females and 136 males, 32 cases were classified as mild, 50 cases were

Table 2 Levels of sCD138 and time frame of sample collection in patients with HFRS

\begin{tabular}{|c|c|c|c|c|c|}
\hline & $\begin{array}{l}\text { Mild group } \\
(n=32)\end{array}$ & $\begin{array}{l}\text { Moderate group } \\
(n=50)\end{array}$ & $\begin{array}{l}\text { Severe group } \\
(n=43)\end{array}$ & $\begin{array}{l}\text { Critical group } \\
(n=51)\end{array}$ & $\begin{array}{l}\text { Control group } \\
(n=35)\end{array}$ \\
\hline \multicolumn{6}{|l|}{ sCD138, ng/mL } \\
\hline a Acute stage & $3.45 \pm 2.32$ & $5.29 \pm 3.64$ & $4.73 \pm 3.83$ & $5.22 \pm 3.19$ & $0.819(0.260)$ \\
\hline b Convalescent stage & $2.71 \pm 2.45^{*}$ & $2.78 \pm 1.39^{*}$ & $2.54 \pm 1.68^{*}$ & $2.56 \pm 2.58^{*}$ & $0.819(0.260)$ \\
\hline \multicolumn{6}{|l|}{ Length of time (days) } \\
\hline${ }^{c}$ Acute stage & $7(4)$ & $7(3)$ & $7(3)$ & $7(4)$ & - \\
\hline${ }^{d}$ Convalescent stage & $11.5(4)$ & $13(3)$ & $17(10)$ & $22(10)$ & - \\
\hline
\end{tabular}

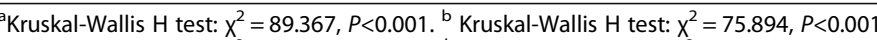

${ }^{c}$ Kruskal-Wallis $\mathrm{H}$ test: $X^{2}=5.612, P=0.312 .{ }^{d}$ Kruskal-Wallis $\mathrm{H}$ test: $X^{2}=45.344, P<0.001$

${ }^{*}$ Comparison of the levels of $\mathrm{sCD} 138$ between the acute and convalescent stage: mild group, $\mathrm{t}=0.873 P=0.387$; moderate group, $\mathrm{t}=4.253 P<0.001$; severe group, $\mathrm{t}=3.253 P=0.002 ;$ critical group, $\mathrm{t}=3.748 P<0.001$ 


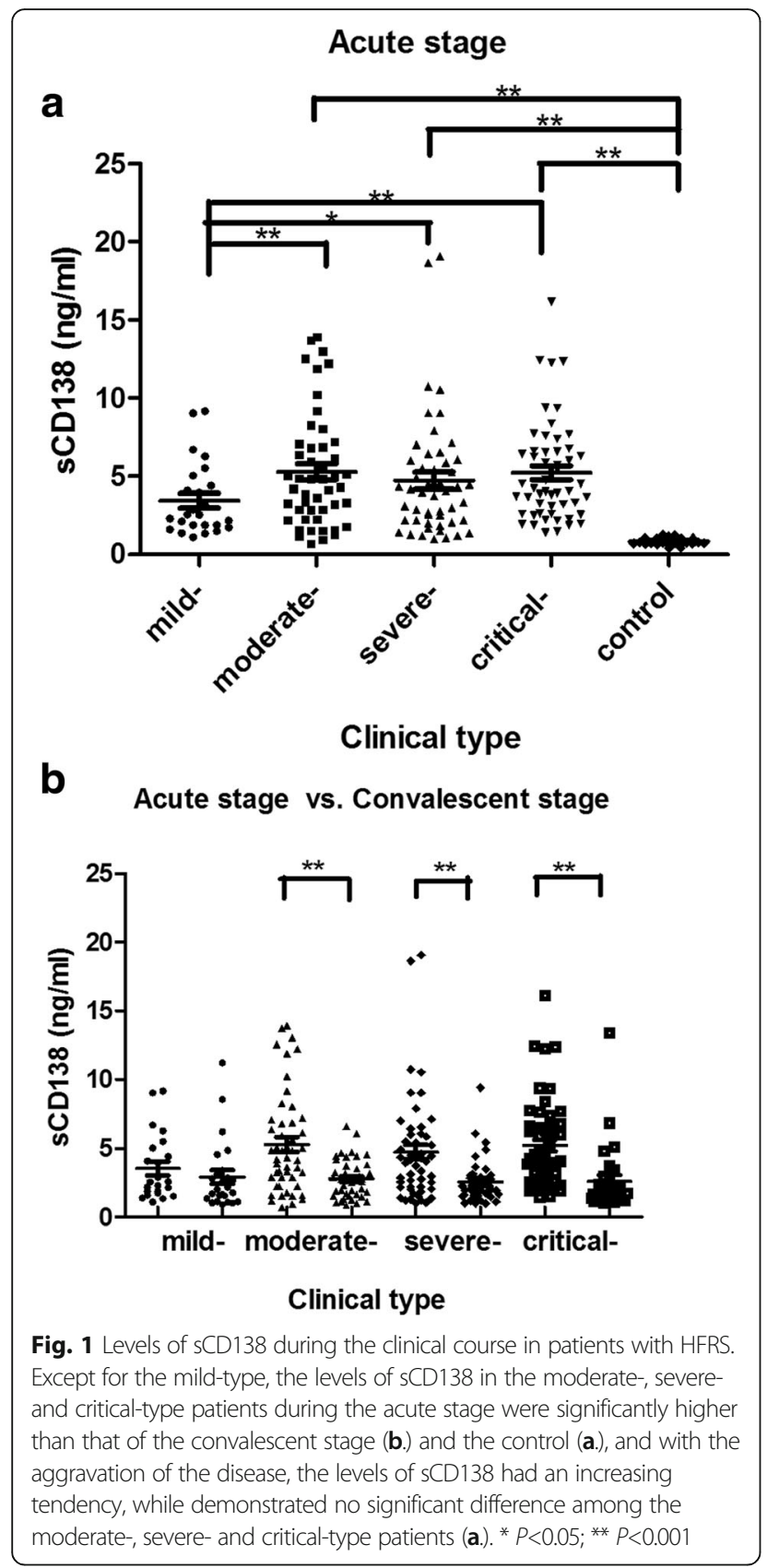

classified as moderate, 43 cases were classified as severe and 51 cases were classified as critical according to the HFRS criteria of clinical classification. Eighteen critical individuals died during the acute stage with fatality rate of $10.23 \%$. There was no significant difference in the sex or age distribution among the groups $(P>0.05)$ (Table 1$)$.

\section{Levels of $\mathrm{sCD} 138$ in patients with HFRS}

The duration from disease onset to sample collection during the acute stage among the four groups was not significantly different $(P=0.312)$ (Table 2$)$. Except for the mild-type, the levels of sCD138 in the moderate-,
Table 3 Spearman's correlation analysis in patients with HFRS

\begin{tabular}{lll}
\hline Variables & \multicolumn{2}{c}{$s$ CD138 } \\
\cline { 2 - 3 } & $r$ & $P$ value \\
\hline WBC & 0.454 & $<0.001$ \\
ALT & 0.185 & 0.001 \\
AST & 0.426 & $<0.001$ \\
GLU & 0.232 & $<0.001$ \\
PT & 0.143 & 0.018 \\
APTT & 0.270 & $<0.001$ \\
Fib & -0.329 & $<0.001$ \\
PLT & -0.433 & $<0.001$ \\
ALB & -0.447 & $<0.001$ \\
BUN & 0.235 & $<0.001$ \\
Scr & 0.138 & $<0.001$
\end{tabular}

Abbreviations: $r$ correlation coefficient, $s C D 138$, soluble CD138; WBC, white blood cells; PLT, platelets; $A L B$, albumin; $A L T$, alanine aminotransferase; $A S T$, aspartate aminotransferase; GLU, glucose; $P T$, prothrombin time; $A P T$, activated partial thromboplastin time; Fib, fibrinogen; $B U N$, blood urea nitrogen; $\mathrm{Cr}$, creatinine

severe- and critical-type patients during the acute stage were significantly higher than that of the convalescent stage and the control $(\mathrm{P}<0.05)$, and with the aggravation of the disease, the levels of sCD138 during the acute stage had an increasing tendency, while demonstrated no significant difference among the moderate-, severeand critical-type patients $(P>0.05)$. The comparison of the sCD138 during the convalescent stage among the four types demonstrated no significant difference $(\mathrm{P}<0.05)$ (Table 2, Fig. 1).

\section{Spearman's correlation analysis and ROC curves}

sCD138 was negatively correlated with Fib, PLT and $\mathrm{ALB}$, and was positively correlated with $\mathrm{WBC}$ and $\mathrm{AST}$, with correlation coefficients above $0.300 \quad(\mathrm{P}<0.05)$ (Table 3, Fig. 2). sCD138 demonstrated predictive value for prognosis with the AUC of $0.778(P<0.001)$ (Table 4, Fig. 3$)$.

\section{Discussion}

As an acute viral disease, HFRS has the basic clinical characteristics of sepsis [20], but it also has a unique pathophysiologic feature. The hypotensive phase of HFRS (e.g., low blood pressure and circulation collapse) usually occurs between day 3 and day 7 of the clinical course, and grave HFRS patients can manifest more severe leukemoid reaction, plasma leakage and coagulation disorders [21, 22] compared with septic patients, which would lead to massive bleeding, profound shock, severe tissue hypoperfusion and severe hypoxia, potentially rendering renal, cardiac, cerebellar and hepatic injury [23, 24]. Although there were some studies demonstrated that $\mathrm{sCD} 138$ can be considered as a novel marker on predicting prognosis in lung injury and sepsis patients [25-27], while as far as we know, until now, 

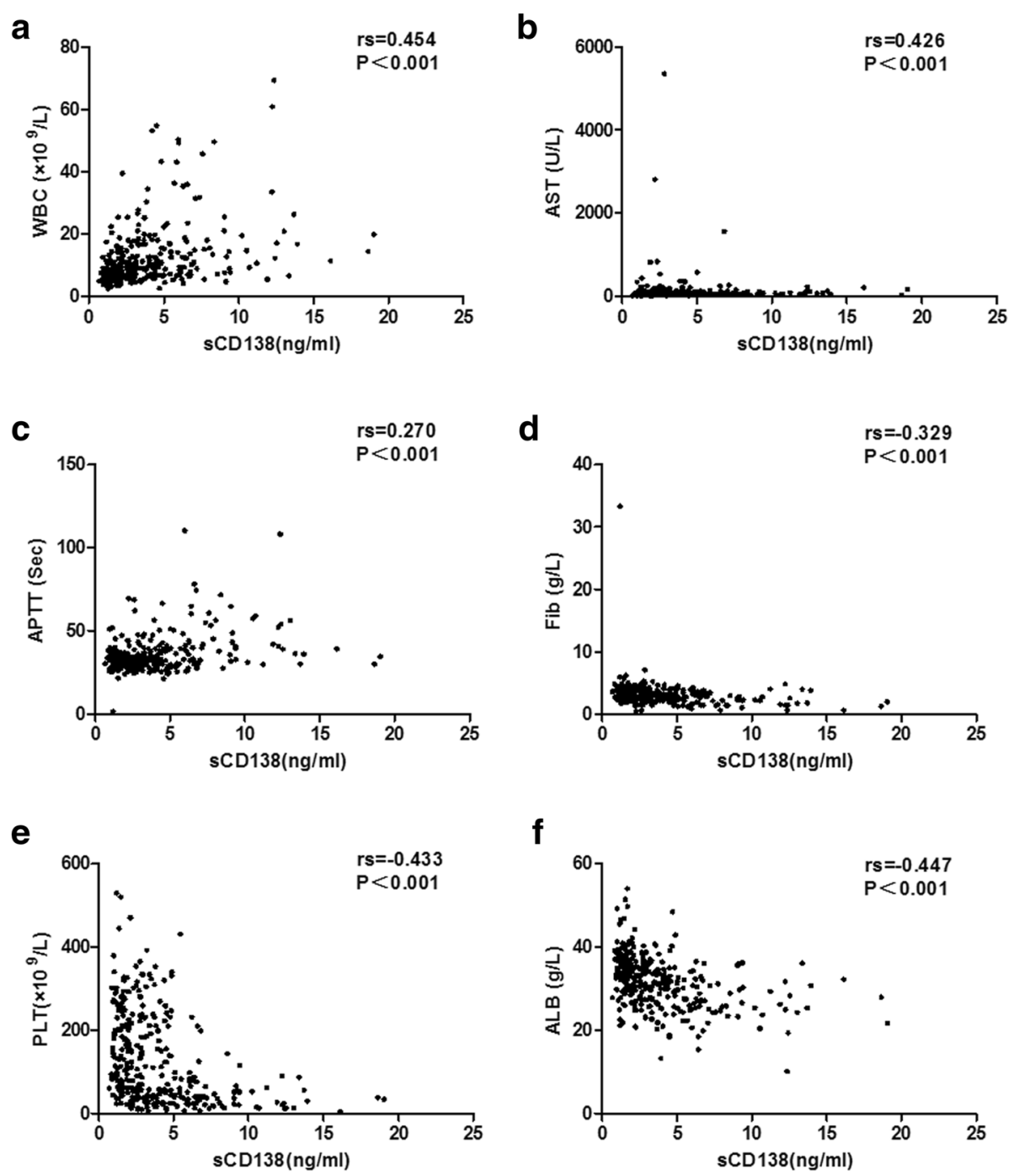

Fig. 2 Correlation between SCD138 and WBC (a.), AST (b.), APTT (c.), Fib (d.), PLT (e.) and ALB (f.) in patients with HFRS by Spearman's correlation analysis. SCD138 was obviously positively correlated with WBC and AST, and was negatively correlated with Fib, PLT and ALB (P<0.05). Abbreviations: sCD138, soluble CD138; WBC, white blood cells; PLT, platelets; ALB, albumin; AST, aspartate aminotransferase; APTT, activated partial thromboplastin time; Fib, fibrinogen

there was no research on exploring its expression and relationship with disease severity and prognosis of HFRS. Research of the expression of plasma sCD138 on different clinical types and courses would be benefit to illustrating its role on pathogenesis, disease severity and prognosis evaluation on HFRS by clinicians.
From this study, we observed that the levels of sCD138 in the moderate-, severe- and critical-type patients during the acute stage were significantly higher than that of the convalescent stage and the control, while the expression of sCD138 demonstrated no significant difference among the three types, which indicates that high levels of sCD138

Table 4 Predictive values for prognosis with sCD138 in patients with HFRS

\begin{tabular}{|c|c|c|c|c|c|c|c|}
\hline \multirow[t]{2}{*}{ Variables } & \multirow[t]{2}{*}{ AUC } & \multirow[t]{2}{*}{ a $p$ value } & \multirow[t]{2}{*}{${ }^{b}$ Cut off value } & \multirow[t]{2}{*}{${ }^{c}$ Sensitivity } & \multirow[t]{2}{*}{${ }^{\text {c }}$ Specificity } & \multicolumn{2}{|c|}{${ }^{\circ} 95 \%$ Cl for AUC } \\
\hline & & & & & & Lower & Upper \\
\hline sCD138 & 0.778 & $<0.001$ & 5.868 & 66.7 & 83.2 & 0.678 & 0.877 \\
\hline
\end{tabular}

Abbreviations: $A U C$, area under the curve; $C l$, confidence interval; $s C D 138$, soluble CD138

${ }^{\text {a }} P$ value for calculated AUC in predicting death

bUnits of the parameters were $\mathrm{ng} / \mathrm{ml}$

'Sensitivity, specificity and $95 \% \mathrm{Cl}$ are all presented as percentages 


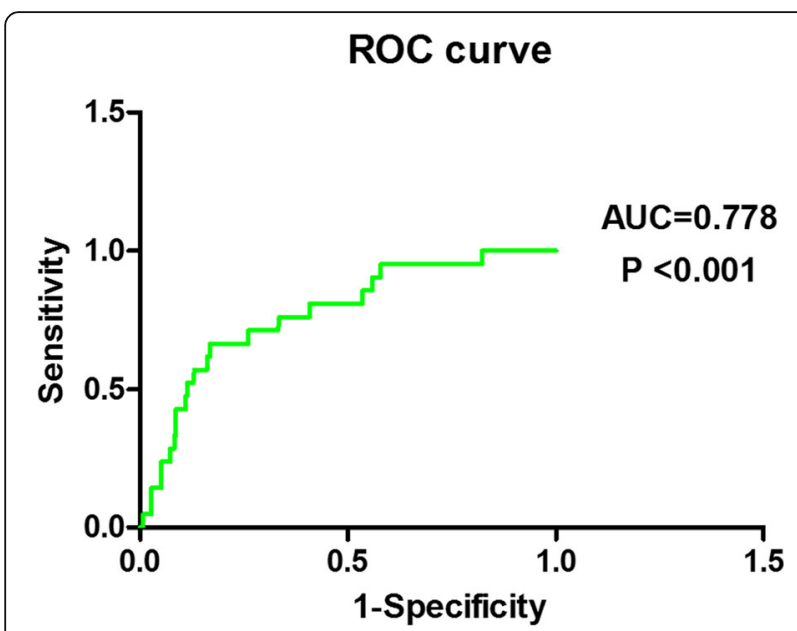

Fig. 3 Predictive values for prognosis with $\mathrm{SCD} 138$ by ROC curve analysis. SCD138 showed predictive value of prognosis, with the AUC of $0.778(P<0.001)$

could reflect the shedding of glycocalyx under systemic inflammatory response to a degree, which was similar with the patients with severe sepsis and septic shock [14, 28].

Our previous study $[3,4,19]$ analyzed the levels of 12 routinely tested laboratory parameters in HFRS patients and demonstrated that WBC, PLT and ALB can be beneficial as early indicators of severity and prognosis in HFRS patients. High WBC can reflect the degree of inflammatory reaction; destruction or dysfunction of PLT (low PLT) [29] and low Fib can reflect the increasing risk of hemorrhage; while the low expression of ALB can represent the result of leakage from the damage vessel to the tissue interspace, which can reflect the loss of vascular integrity and the change of vascular permeability to a degree $[24,30]$. In this study, we observed that sCD138 was obviously positively correlated with WBC, and was negatively correlated with Fib, PLT and ALB $(\mathrm{P}<0.05)$ (Table 3, Fig. 2), which not only provided supporting evidence on the major origin of sCD138 coming from the shedding of the PGs, but also illustrated that the destruction of GCX usually companied with more reactive inflammatory and immunity response, permeability change of vascular and clotting dysfunction.

In this study we also observed that plasma sCD138 were positively correlated with AST. Considering the high level of AST can manifest the abnormality of heart and liver function [4.19], which further indicated that the expression of plasma $\mathrm{sCD} 138$ can reflect the injury of multi-organ system and disease severity to a degree. Furthermore, in our study, the ROC analysis demonstrated that SCD138 showed predictive value of prognosis, with the AUC of $0.778(P<0.001)$, which indicated that $\mathrm{SCD} 138$ had better predictive capacity for prognosis of HFRS and potential clinical application, like WBC, AST, PLT, ALB and HMGB-1 explored from our previous studies $[2-4,19]$.

As an observational prospective study, we got a meaningful conclusion that dynamic detection of plasma sCD138 might be benefit to evaluating the disease severity and prognosis of the HFRS patients, while there were still some limitations:

First, this study was conducted at a single center for infectious diseases. The length of time from collection of the blood samples was not unified or precise, considering the different clinical conditions and phases on admission in our center, and we can only define two periods: the acute and the convalescent stages. Although there was no significant difference in the median collection time of the samples in the acute stage, the dynamic change of the levels of plasma sCD138 can also be influenced by this variation and the pathologic inter-patient variability during the acute phase of infection.

Second, as we mentioned above, the relatively small number of cases and also the fragmentation of data collection made the statistical power small relatively. In this study, we were not able to collect more cases of HFRS to enlarge study samples in the last 3 years because of the lower incidence of HFRS in our region. Analyzing the major reasons, the interval from 2011 to 2013 was a period of city economy development, and the real estates construction led to destroyed ecological environment usually accompanied with the destruction of the rodents' life cycle and environment, which cause a large numbers of rodents, especially the Apodemus agrarius, major source of infection, immigrate to the colonized region of the human and cause infection; furthermore, the negligence of HFRS vaccine injection in time on that interval was another reason. In the last 3 years, with the caution of local epidemiological investigation and active immunity of HFRS vaccine injection on population with inoculation rate above $85 \%$, the HFRS incidence was obviously low. While, we should also be pay attention to the next large epidemic interval of HFRS in this region considering the descending valence of vaccine (usually sustain $3-5$ years).

Furthermore, only 18 critical individuals died were enrolled in this study, the relative small number enrolled would influence the result of the ROC curve analysis. Finally, the clinical outcomes and classifications of the HFRS patients might be biased due to the lack of a more standardized protocol for the management of patients with HFRS until now.

\section{Conclusion}

Dynamic detection of plasma sCD138 might be benefit to evaluating the disease severity and prognosis of the patients with HFRS. 


\section{Abbreviations}

AKI: Acute kidney injury; ALB: Albumin; ALT: Alanine aminotransferase; APTT: Activated partial thromboplastin time; ARDS: Acute respiratory distress syndrome; AST: Aspartate aminotransferase; AUC: Area under the ROC curve; BUN: Blood urea nitrogen; Cl: Confidence interval; EDTA: Ethylenediamine tetraacetic acid; ELISA: Enzyme linked immunosorbent assay; ESL: Endothelial surface layer; Fib: Fibrinogen; GCX: Glycocalyx; GLU: Glucose; HFRS: Hemorrhagic fever with renal syndrome; HTNV: Hantaan virus; MODS: Multiple organ dysfunction syndrome; PGs: Proteoglycans; PLT: Platelets; PT: Prothrombin time; ROC: Receiver operating characteristic; SCD138: Soluble CD138; Scr: Serum creatinine; SIRS: Systemic inflammatory response syndrome; WBC: White blood cells

\section{Acknowledgements}

We acknowledge the support of Jianqi Lian and Lin-Xu Wang in data management.

\section{Funding}

This work was supported by the Basic and Clinical Research Projects of Technology Innovation Development Foundation in Tangdu Hospital (NO.2016JCYJ001; NO.2015LCYJ017), the Technology Development Foundation of the Fourth Military Medical University (NO.2016XD252), the National Natural Science Foundation of China (No.81373118) and the National Basic Research Program of China (973 Program) (No.2012CB518905).

\section{Authors' contribution}

$H D, X F B$ and PZW conceived and designed the experiments. JL, HD and $X Y W$ analyzed the data. $J$, HD and $H J$ wrote the paper. $J$, HD and $Y Z$ reviewed and collected the data. $\mathrm{XFB}, \mathrm{HJ}$ and $\mathrm{PZW}$ reviewed the manuscript. All authors read and approved the final manuscript.

\section{Availability of data and materials}

Data contains personal information. The data will not be made available in order to protect the participants' identity.

\section{Ethics approval and consent to participate}

The study was approved by the Institutional Review Board of Tangdu Hospital. Before inclusion, the patients were informed about the objectives of this study, and they or their direct relatives agreed and signed the informed consent form before inclusion.

\section{Consent for publication}

Not applicable.

\section{Competing interests}

The authors declare that they have no competing interests.

\section{Publisher's Note}

Springer Nature remains neutral with regard to jurisdictional claims in published maps and institutional affiliations.

\section{Received: 10 September 2017 Accepted: 21 February 2018}

\section{Published online: 01 March 2018}

\section{References}

1. Jonsson $C B$, Figueiredo LT, Vapalahti O. A global perspective on hantavirus ecology, epidemiology, and disease. Clin Microbiol Rev. 2010;23(2):412-41.

2. Du H, Li J, Yu H, Lian J, Zhang Y, Zhang Y, Bai X, Wang P. HMGB-1 as a novel predictor of disease severity and prognosis in patients with hemorrhagic fever with renal syndrome. Mediat Inflamm. 2015;2015:696248.

3. Du H, Li J, Yu HT, Jiang W, Zhang Y, Wang JN, Wang PZ, Bai XF. Early indicators of severity and construction of a risk model for prognosis based upon laboratory parameters in patients with hemorrhagic fever with renal syndrome. Clin Chem Lab Med. 2014;52(11):1667-75.

4. Du H, Li J, Jiang W, Yu H, Zhang Y, Wang J, Wang P, Bai X. Clinical study of critical patients with hemorrhagic fever with renal syndrome complicated by acute respiratory distress syndrome. PLoS One. 2014;9(2):e89740.

5. Jiang $H$, Zheng XY, Wang LM, Du H, Wang PZ, Bai XF. Hantavirus infection: a global zoonosis problem. Virol Sin. Published online: 23 January 2017. DOl: 10.1007/s12250-016-3899-x

6. Jiang $H$, Du H, Wang LM, Wang PZ, Bai XF. Hemorrhagic fever with renal syndrome: pathogenesis and clinical picture. Front Cell Infect Microbiol. 2016;6:1.
7. Du H, Bai X, Lian J, Li J, Zhang Y, Wang P, Jiang H. Changes in plasma adiponectin concentrations in patients with hemorrhagic fever with renal syndrome: an observational prospective study. Medicine (Baltimore). 2016;95(6):e2700.

8. Alphonsus CS, Rodseth RN. The endothelial glycocalyx: a review of the vascular barrier. Anaesthesia. 2014;69:777-84.

9. Radeva MY, Waschke J. Mind the gap: mechanisms regulating the endothelial barrier. Acta Physiol (Oxf). 2017; https://doi.org/10.1111/apha. 12860. [Epub ahead of print].

10. Dane MJ, van den Berg BM, Lee DH, Boels MG, Tiemeier GL, Avramut MC, van Zonneveld AJ, van der Vlaq J, Vink H, Rabelink TJ. A microscopic view on the renal endothelial glycocalyx. Am J Physiol Renal Physiol. 2015;308(9):F956-66.

11. Ushiyama A, Kataoka H, Lijima T. Glycocalyx and its involvement in clinical pathophysiologies. J Intensive Care. 2016:4(1):59.

12. Reitsma S, Slaaf DW, Vink H, van Zandvoort MA, oude Eqbrink MG. The endothelial glycocalyx: composition, functions, and visualization. Pflugers Arch. 2007;454(3):345-59.

13. Page $A V$, Liles WC. Biomarkers of endothelial activation/dysfunction in infectious diseases. Virulence. 2013;4(6):507-16.

14. Chelazzi C, Villa G, Mancinelli P, De Gaudio AR, Adembri C. Glycocalyx and sepsis-induced alterations in vascular permeability. Crit Care. 2015;19:26.

15. Bruegger D, Brettner F, Rossberg I, Nussbaum C, Kowalski C, Januszewska K, Becker BF, Chappell D. Acute degradation of the endothelial glycocalyx in infants undergoing cardiac surgical procedures. Ann Thorac Surg. 2015; 99(3):926-31.

16. Neves FM, Meneses GC, Sousa NE, Menezes RR, Parahyba MC, Martins AM, Libório AB. Syndecan-1 in acute decompensated heart failure-association with renal function and mortality. Circ J. 2015;79(7):1511-9.

17. Tarbell JM, Cancel JM. The glycocalyx and its significance in human medicine. J Intern Med. 2016;280(1):97-113.

18. Johansson PI, Stensballe J, Rasmussen LS, Ostrowskj SR. A high admission syndecan-1 level, a marker of endothelial glycocalyx degradation, is associated with inflammation, protein $C$ depletion, fibrinolysis, and increased mortality in trauma patients. Ann Surg. 2011;254(2):194-200.

19. Du H, Wang PZ, Li J, Bai L, Li H, Yu HT, Jiang W, Zhang Y, Wang JN, Bai XF. Clinical characteristics and outcomes in critical patients with hemorrhagic fever with renal syndrome. BMC Infect Dis. 2014;14:191.

20. Singer M, Deutschman CS, Seymour CW, Shankar-Hari M, Annane D, Bauer M, Bellomo R, Bernard GR, Chiche JD, Coopersmith CM, Hotchkiss RS, Levy MM, Marshall JC, Martin GS, Opal SM, Rubenfeld GD, van der Poll T, Vincent $J \mathrm{~L}$, Angus DC. The third international consensus definitions for sepsis and septic shock (sepsis-3). JAMA. 2016;315(8):801-10.

21. Rasche FM, Uhel B, Krüger DH, Karges W, Czock D, Hampl W, Keller F, Meisel $\mathrm{H}$, von Müller L. Thrombocytopenia and acute renal failure in Puumala hantavirus infections. Emerg Infect Dis. 2004:10(8):1420-5.

22. Bae JS. Effects of lower concentration thrombin on high-mobility group box 1 protein-mediated inflammatory responses. Inflammation. 2011:35(3):1078-86.

23. Kim S, Sung SH, An HR, Jun YH, Yu M, Ryu DR, Kim SJ, Kang DH, Choi KB. A case report of crescentic glomerulonephritis associated with hantaan virus infection. Nephrol Dial Transplant. 2010;25(8):2790-2.

24. Kim YO, Yoon SA, Ku YM, Yang CW, Kim YS, Kim SY, Choi EJ, Chang YS, Bang BK. Serum albumin level correlates with disease severity in patients with hemorrhagic fever with renal syndrome. J Korean Med Sci. 2003:18(5):696-700.

25. Schmidt EP, Yang Y, Janssen WJ, Gandjeva A, Perez MJ, Barthel Z, Zemans RL, Bowman JC, Koyanaqi DE, Yunt ZX, Smith LP, Cheng SS, Overdier KH, Thompson KR, Geraci MW, Douglas IS, Pearse DB, Tuder RM. The pulmonary endothelial glycocalyx regulates neutrophil adhesion and during experimental sepsis. Nat Med. 2012;18(8):1217-23.

26. Sallisamli M, Tenhunen J, Yang R, Oksala N, Pettilä V. Vascular adhesion protein-1 and syndecan-1 in septic shock. Acta Anaesthesiol Scand. 2012; 56(3):316-22.

27. Ostrowski SR, Berg RM, Windeløv NA, Meyer MA, Plovsing RR, Møller K, Johansson PI. Coagulopathy, catecholamines, and biomarkers of endothelial damage in experimental human endotoxemia and in patients with severe sepsis: a prospective study. J Crit Care. 2013;28(5):586-96.

28. Steppan J, Hofer S, Funke B, Brenner T, Henrich M, Martin E, Weitz J, Hofmann U, Weigand MA. Sepsis and major abdominal surgery lead to flaking of the endothelial glycocalix. J Surg Res. 2011;165(1):136-41.

29. Levi M, Löwenberg EC. Thrombocytopenia in critically ill patients. Semin Thromb Hemost. 2008;34(5):417-24

30. Cosgriff TM, Lewis RM. Mechanisms of disease in hemorrhagic fever with renal syndrome. Kidney Int. 1991;40(35):72-9. 